\title{
Traumatic sacral dermoid cyst rupture with intracranial subarachnoid seeding of lipid particles: illustrative case
}

\author{
*Alexander Perdomo-Pantoja, MD, Hesham Mostafa Zakaria, MD, Brendan F. Judy, MD, Jawad M. Khalifeh, MD, Jose L. Porras, MD, \\ Tej D. Azad, MD, MS, Brian Y. Hwang, MD, Timothy F. Witham, MD, Chetan Bettegowda, MD, PhD, and Nicholas Theodore, MD \\ Department of Neurosurgery, Johns Hopkins University School of Medicine, Baltimore, Maryland
}

BACKGROUND Intracranial deposits of fat droplets are an unusual presentation of a spinal dermoid cyst after spontaneous rupture and are even more uncommon after trauma. Here, the authors present a case with this rare clinical presentation, along with a systematic review of the literature to guide decision making in these patients.

OBSERVATIONS A 54-year-old woman with Lynch syndrome presented with severe headache and sacrococcygeal pain after a traumatic fall. Computed tomography of the head revealed multifocal intraventricular and intracisternal fat deposits, which were confirmed by magnetic resonance imaging (MRI) of the neuroaxis; in addition, a ruptured multiloculated cyst was identified within the sacral canal with proteinaceous/hemorrhagic debris, most consistent with a sacral dermoid cyst with rupture into the cerebrospinal fluid (CSF) space. An unruptured sacral cyst was later noted on numerous previous MRI scans. In our systematic review, we identified 20 similar cases, most of which favored surgical treatment.

LESSONS Rupture of an intraspinal dermoid cyst must be considered when intracranial fat deposits are found in the context of cauda equina syndrome, meningism, or hydrocephalus. Complete tumor removal with close postoperative follow-up is recommended to decrease the risk of complications. CSF diversion must be prioritized if life-threatening hydrocephalus is present.

https://thejns.org/doi/abs/10.3171/CASE21355

KEYWORDS cyst rupture; fatty deposits; intraspinal dermoid; intraventricular and subarachnoid spaces

Dermoid cysts are benign congenital tumors that arise when ectodermal elements become trapped within the neural groove at the time of closure during the first weeks of embryonic life. ${ }^{1}$ They comprise $<2 \%$ of tumors of the central nervous system and about $1 \%$ of all spinal tumors. $^{1-3}$ Most spinal dermoid cysts are located in the lumbosacral region ( $\sim 60 \%$ of cases), with a few cases reported in the cervical and thoracic spine. ${ }^{4,5}$ Because of their slow-growing nature, spinal dermoid cysts are usually benign and asymptomatic. However, these tumors can cause symptoms by 2 different mechanisms: They may cause mass effect on adjacent neural structures, or they may rupture.

A dermoid cyst rupture may occur spontaneously, or it may occur after trauma or surgery. ${ }^{6}$ Upon rupture, these cysts can cause various symptoms due to release of the cyst's content into the subarachnoid space, inducing chemical aseptic meningitis. Although the rupture of spinal dermoids is well known, ${ }^{7,8}$ only a few cases have actually documented distant intracranial fat deposits. ${ }^{6,9-23}$ Here, we illustrate an uncommon presentation of a sacral dermoid cyst. After traumatic rupture, it released fat droplets into the lumbar cistern. These fat droplets are less dense than cerebrospinal fluid (CSF), so they traveled caudally into the cranial subarachnoid and intraventricular spaces and seeded. We also performed a systematic review of the literature to identify similar cases and offer guidance on the decision-making process in this unusual scenario.

\footnotetext{
ABBREVIATIONS CSF = cerebrospinal fluid; $C T$ = computed tomography; $M R I=$ magnetic resonance imaging INCLUDE WHEN CITING Published December 13, 2021; DOI: 10.3171/CASE21355.

SUBMITTED June 13, 2021. ACCEPTED July 19, 2021.

* A.P.P. and H.M.Z. contributed equally to this work.

(c) 2021 The authors, CC BY-NC-ND 4.0 (http://creativecommons.org/licenses/by-nc-nd/4.0/).
} 


\section{Illustrative Case \\ Clinical Findings}

For this case report, we followed the consensus-based clinical case reporting guideline development guidelines. ${ }^{24} \mathrm{~A} 54-$ year-old woman presented to the emergency department with head injury, without loss of consciousness, and with significant headache, neck rigidity, and tailbone pain after falling backward while rollerblading. Physical examination showed a head contusion with a $3-\mathrm{cm}$ linear laceration to the posterior scalp and coccygeal tenderness. She had a history of Lynch syndrome with multiple neoplasms (i.e., recurrent endometrial cancer, colon cancer, pancreatic neuroendocrine tumor), multiple surgical procedures (i.e., total abdominal hysterectomy, partial colectomy, distal pancreatectomy), and pelvic radiotherapy 1 year before her fall. Due to her oncological history, she had a library of previous imaging that provided comparison studies. After evaluation, imaging work-up, and observation for 48 hours, she was discharged to be seen in follow-up in the clinic.

\section{Imaging Studies}

During her stay in the emergency department, the patient underwent imaging work-up. Radiographs showed no evidence of acute fracture involving the sacrum or coccyx. Computed tomography (CT) of the head was performed due to her severe headache and cranial trauma; this imaging revealed multiple foci of intraventricular, intracisternal fat, which was not present on positron emission tomography/CT 1 month earlier. No evidence of acute intracranial hemorrhage or hydrocephalus was seen. Magnetic resonance imaging (MRI) of the brain demonstrated multifocal fat lobules within the lateral ventricles and the basal cisterns (suprasellar, quadrigeminal, and cerebellopontine angles; Fig. 1). A lumbosacral spine MRI scan showed a multiloculated cystic lesion within the sacral canal with fatty components and layering of proteinaceous/hemorrhagic debris. These features suggested a partial rupture of a sacral dermoid cyst with communication into the adjacent CSF space of the distal lumbosacral thecal sac, explaining the source of the fatty emboli. Comparison MRI scans of the abdomen/pelvis from 1 year earlier revealed a fatty cyst within the sacral canal (Fig. 2).
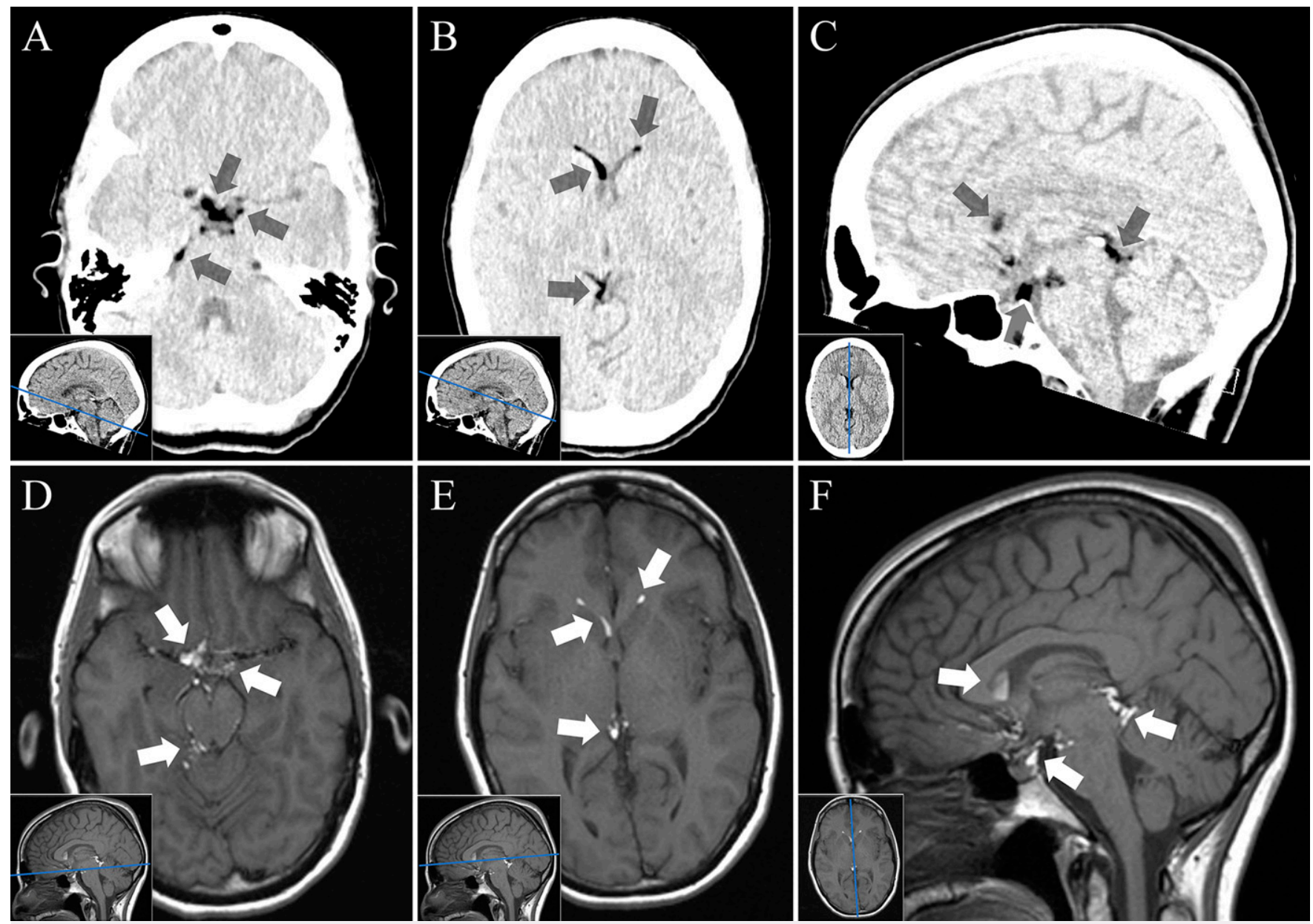

FIG. 1. Axial (A and B) and sagittal (C) cranial CT scans show multifocal intraventricular and intracisternal fat (gray arrows), which appeared new compared with positron emission tomography/CT performed 1 year before the patient's presentation at our institution. Axial $(\mathbf{D}$ and $E)$ and sagittal $(\mathbf{F})$ MRI scans of the brain confirmed the presence of multifocal fat lobules within the nondependent aspects of the lateral ventricles and the basal cisterns (white arrows). A right parietal scalp hematoma was observed, with no acute intracranial hemorrhage or depressed skull fracture. Ventricles, sulci, and basal cisterns were within normal limits. 

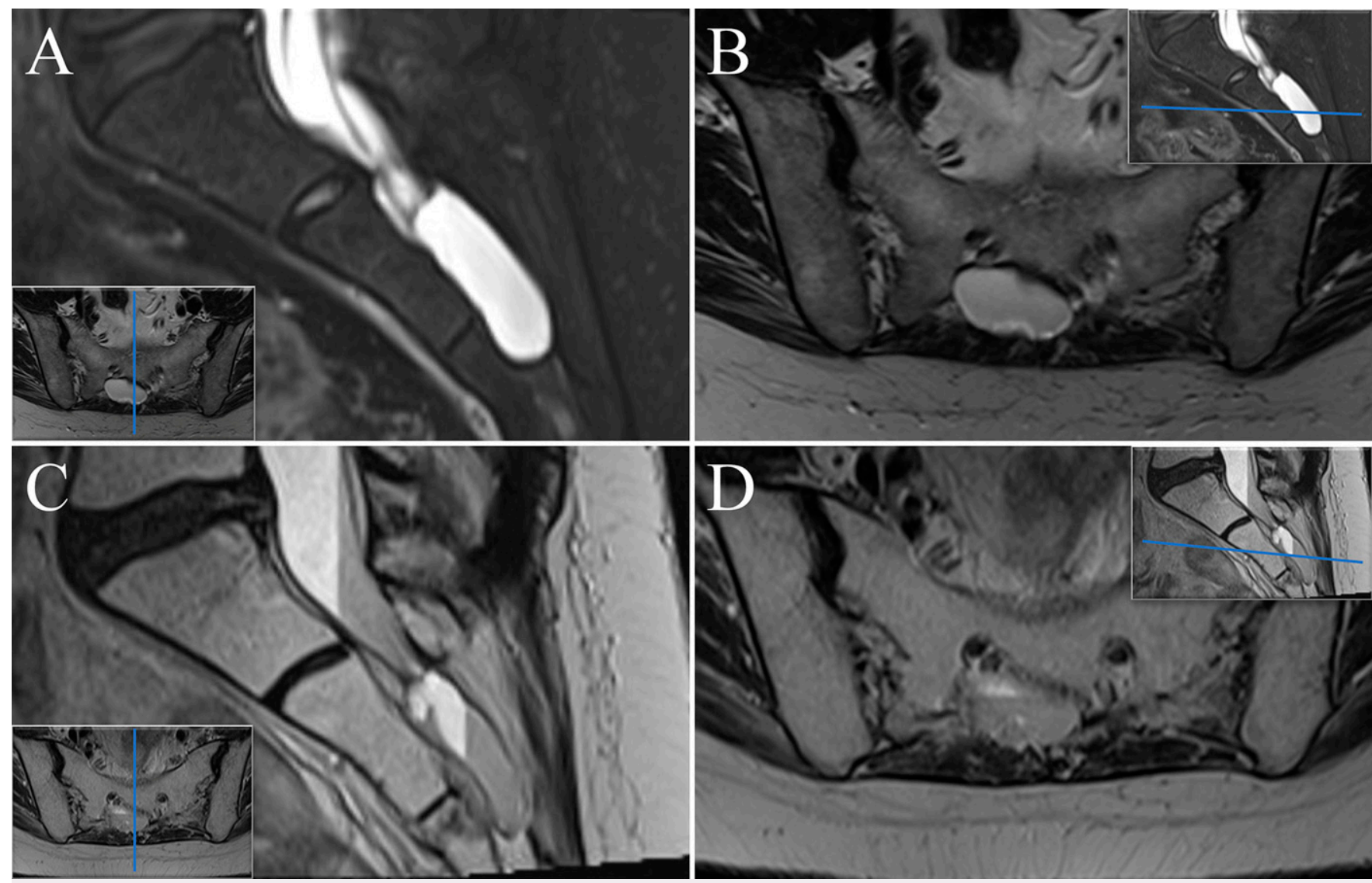

FIG. 2. Sagittal (A) and axial (B) MRI scans from 1 year before presentation showed a sacral lesion without evidence of rupture or hemorrhage. On this admission, sagittal (C) and axial (D) MRI scans of the sacral spine revealed an intrathecal multilobulated cystic lesion centered within the central/right paracentral sacral canal at S2-3. The lesion demonstrated few foci of nodular T1 hyperintensity with subsequent saturation on short tau inversion recovery images, consistent with fatty components. Layering fluid-fluid level within the lesion was consistent with proteinaceous/hemorrhagic debris. The presence of additional layering fluid-fluid level within the distal thecal sac of the lumbosacral junction was consistent with the CSF space's communication.

\section{Management and Follow-Up}

The patient was admitted for observation and pain management, and a repeat brain MRI scan revealed stability of the intracranial fat lesions. After these evaluations, the patient was discharged to continue outpatient follow-up. At her 1-month follow-up visit, resection of the sacral dermoid cyst was recommended due to the risk of further intracranial fat seeding causing hydrocephalus and/or meningism. However, the patient decided not to undergo surgery. One month after discharge, a third brain MRI scan demonstrated more prominent fat lobules within the interhemispheric and sylvian fissures, suggesting redistribution of the fat without evidence of hydrocephalus (Fig. $3 A-C$ ). Six months later, the patient reported symptom improvement, with occasional headaches and mild low back pain. Her last brain MRI revealed a slight decrease in the fat disseminated within the CSF spaces and no signs of hydrocephalus (Fig. 3D-F).

\section{Discussion}

\section{Web-Based Literature Search}

We performed a comprehensive review of the literature in compliance with PRISMA (Preferred Reporting Items for Systematic Reviews and Meta-Analyses) guidelines. ${ }^{25}$ English-language clinical articles published from January 1, 1980, to February 1, 2021, were considered eligible (Fig. 4). The search strategy was planned with the help of a library clinical informationist at our institution, who performed the search based on the PRESS (Peer Review of Electronic Search Strategies) guidelines ${ }^{26}$ using the following databases: PubMed, Embase, Cochrane Library, CINAHL (Cumulative Index to Nursing and Allied Health Literature), Web of Science, Scopus, and ClinicalTrials.gov. The obtained references were imported to the Covidence platform (www.covidence.org) and underwent 2-stage screening for study relevance by 3 independent reviewers (A.P.P., H.M.Z., and J.M.K.), first by title and abstract and then by full text. At the end of each screening stage, any discordance between the reviewers on which articles to include was resolved by the senior author (N.T.). After article selection, we proceeded with data extraction. Information on demographic data, clinical findings, spinal cyst location, and management was collected. Inclusion criteria included peer-reviewed articles reporting cases of intracranial fat seeding secondary to rupture of spinal dermoid cyst. Commentaries, textbooks, expert opinions, letters to the editor, protocols, abstracts, animal studies, and papers with full text unavailable or missing clinical or imaging data of interest were excluded.

\section{Observations}

Since 1992, when Barsi et al. ${ }^{9}$ published the case of a 27 -yearold woman with a thoracolumbar spinal dermoid with leakage of fat 

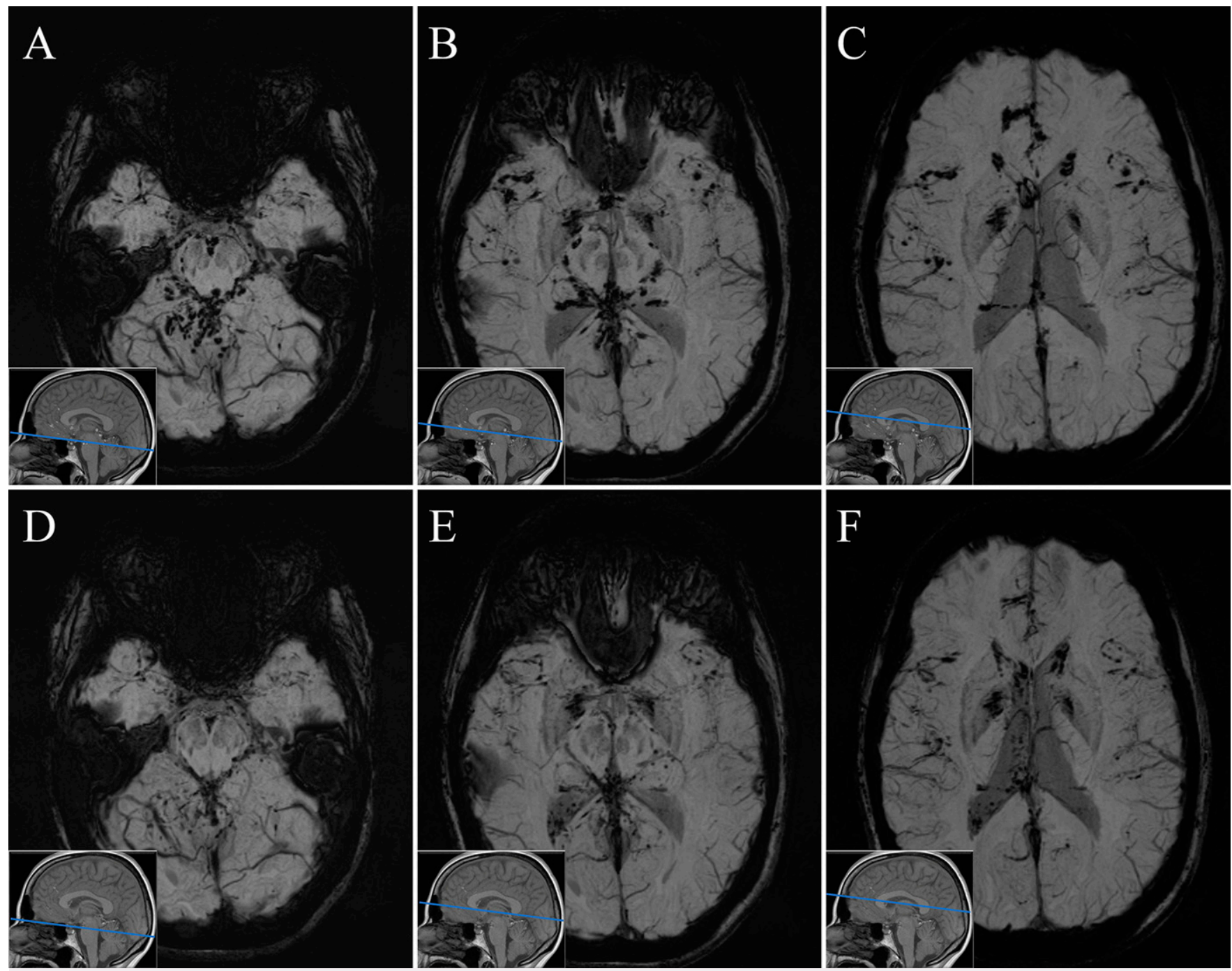

FIG. 3. Axial cranial MRI scans showed a mild increase in the overall burden of foci of fat disseminated within the ventricular system and additional CSF spaces 1 month after patient's fall. Specifically, there were now foci of fat over the cerebellum (A) and within the sylvian fissures (B) and interhemispheric fissure anteriorly $(\mathbf{C})$. These findings represented redistribution, with decreased volume of the focal lipid signal seen in the right side of the suprasellar cistern on the previous MRI scan. Nondependent foci of fat within the right more than the left lateral ventricles and extending throughout the basal cisterns were still observed, without hydrocephalus. Six-month follow-up MRI revealed a slight decrease in prominence of fat lobules disseminated throughout the CSF spaces, which may reflect continued breakdown of larger fat lobules, with overall similar distribution of the fat in basal cisterns over the cerebellum (D) and within the sylvian $(\mathbf{E})$ and interhemispheric $(\mathbf{F})$ fissures. The ventricular system remained unchanged in size and configuration.

droplets into the intracranial CSF spaces, there have been few reports of rupture of a spinal dermoid cyst with intracranial fat migration. Due to the limited number of these cases in the literature and the low incidence of this unusual presentation, no consensus yet exists regarding what treatment should be offered to these patients. To address this gap, our study included the first systematic review analyzing all published case reports to date that describe this clinical scenario. After nearly 700 references were systematically reviewed, only 16 articles $^{6,9-23}$ with 20 patients were identified and provided firsthand information on demographics, characteristics of dermoid cysts, and symptom presentation, resulting in a total of 17 articles with 21 cases when the present study is included (Table 1).

All 21 patients were adults; the mean age was 41.1 (standard deviation, 17.8 ) years, with a strong male predominance (7:1 ratio).
The spinal dermoid cysts were mostly located in the lumbar spine (11 of $21[52.4 \%]$ ); others were located in the thoracolumbar (5 of $21[23.8 \%]$ ), sacral (3 of $21[14.3 \%])$, thoracic (1 of $21[4.8 \%])$, and lumbosacral regions (1 of 21 [4.8\%]). Although most dermoid cysts were congenital (17 of 21 [81.0\%]), we identified 4 cases $(19.0 \%)$ that were presumably postsurgical in patients with a history of a lumbar procedure at a young age or years before symptom onset. In all cases previously reported, except for that reported by Scearce et al., ${ }^{21}$ the symptoms followed the spontaneous rupture of the dermoid cyst. The present case is the first since 1993 to document the rare presentation of rupture after trauma and is made even rarer with the associated subarachnoid seeding. The wide spectrum of presenting symptoms in these patients can be grouped into 3 main categories: (1) cauda equina syndrome, (2) meningism, and (3) 


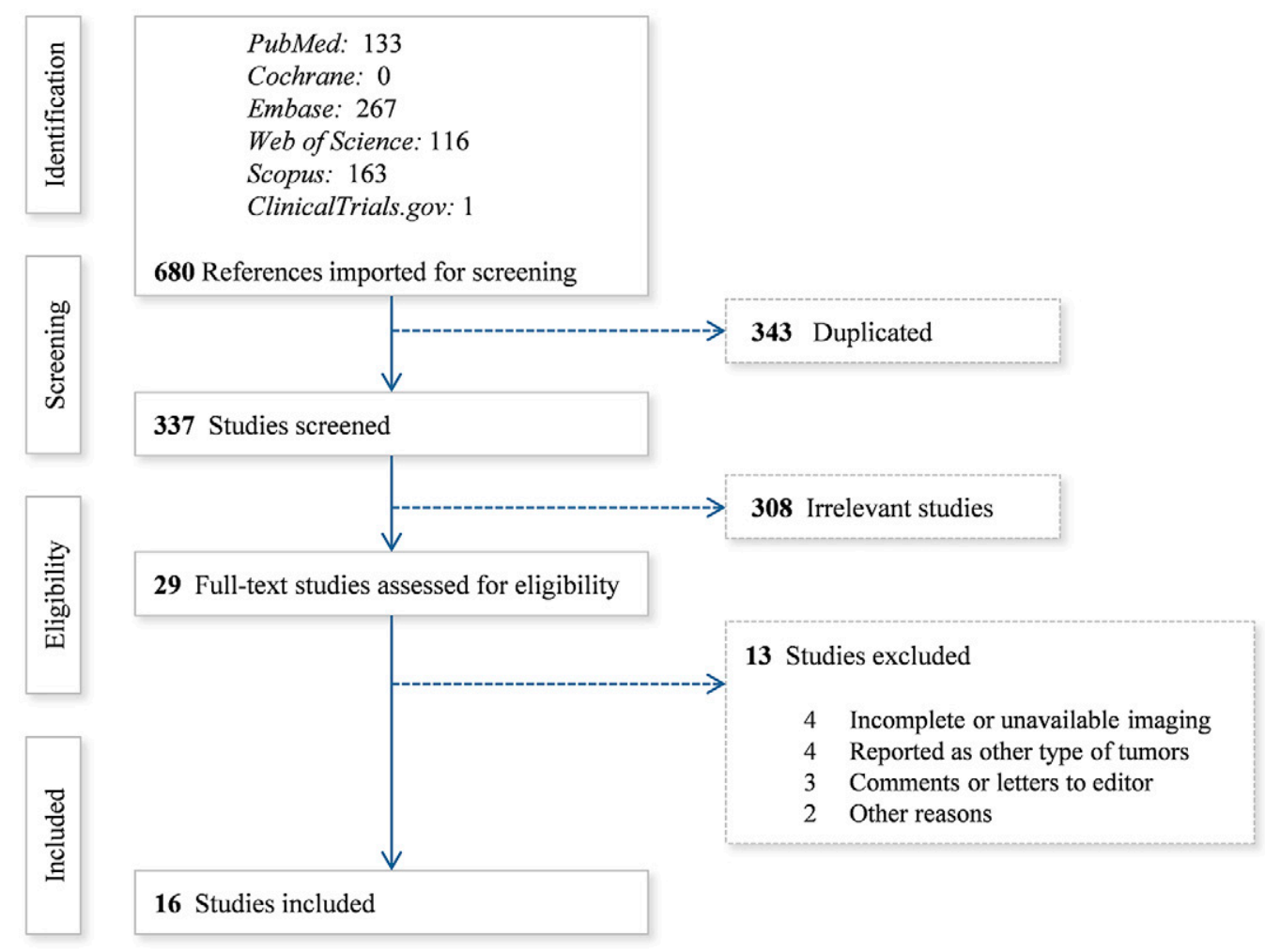

FIG. 4. PRISMA workflow for our systematic review.

hydrocephalus. Most patients had progressive cauda equina symptoms (14 of 21 [66.7\%]); fewer had meningism (9 of 21 [42.9\%]) or both cauda equina symptoms and meningism (3 of 21 [14.3\%]). In addition, several patients developed hydrocephalus (5 of 21 [23.8\%]) and had signs and symptoms of intracranial hypertension at some point.

In general terms, the various symptoms produced by spinal dermoid cysts can be attributed to 2 mechanisms. The first mechanism considers the tumor's mass effect, and signs are dependent on the tumor location, consistency, growth rate, and extension of the involvement of neural structures. This mechanism is the more common of the 2 and is responsible for some of the cauda equina symptoms experienced by these patients, such as low back and radicular pain, lower extremity weakness and sensory disturbances, and sphincter dysfunction. The second mechanism presumes rupture of the spinal dermoid cyst, which empties its content into the CSF space or within the central canal. It has been hypothesized that the breakdown of keratin to cholesterol can cause an inflammatory response, leading to arachnoiditis and ventriculitis with mental decline or even obstructive hydrocephalus. ${ }^{15}$ This chemical meningitis-like reaction can explain meningism symptoms such as severe headache, nuchal rigidity, and seizures. In addition, the rupture of a spinal dermoid cyst is also linked to the migration of fatty droplets through the CSF. Although these fatty particles are detected more often in the subarachnoid space surrounding the spinal cord and inside the central spinal canal, they can travel intracranially and block the CSF flow within the ventricular system, particularly at the level of the aqueduct of Sylvius, resulting in triventricular hydrocephalus.

Management of a spinal dermoid cyst depends on the clinical presentation of each case, and it must target the underlying mechanism responsible for the patient's symptoms. In patients complaining of cauda equina symptoms, spinal canal decompression with tumor resection is the treatment of choice. It relieves neural impingement by removing mechanical pressure on structures inside the spinal canal and functions as a preventive treatment against spontaneous dermoid cyst rupture. Among the cases reported, surgical removal was performed in $71.4 \%$ (10 of 14) of patients with cauda equina symptoms, whereas 1 patient died preoperatively due to a massive pulmonary embolus. ${ }^{21}$ The management modality was unspecified in the remaining patients with cauda equina symptoms. When a rupture has already occurred or there is evidence of recurrence after previous removal surgery, a complete tumor resection should still be considered because the risk of rerupture-and subsequent complications-remains. For instance, $40 \%$ (2 of 5 ) of the patients who developed hydrocephalus had a history of dermoid cyst resection years before, highlighting the importance of complete resection and close follow-up to confer benefit over conservative management. Still, observation was adopted in $4(19.0 \%)$ of all 21 cases, including ours. It is noteworthy that none of these patients exhibited signs or symptoms of cauda equina syndrome, whereas 3 complained of meningism and 1 presented with acute hydrocephalus that was treated with ventriculostomy. In the present case, a removal procedure was offered; however, the patient decided to avoid surgery and undergo close follow-up. On the basis of these reports, we consider that observation can still be an option in selected cases when compression signs are absent, meningism is being managed, and hydrocephalus - if present-has been resolved.

Management for patients who present with meningism has been significantly underreported. Of the 9 cases presenting with meningism included in this review, only 2 patients (including ours) reported the use 


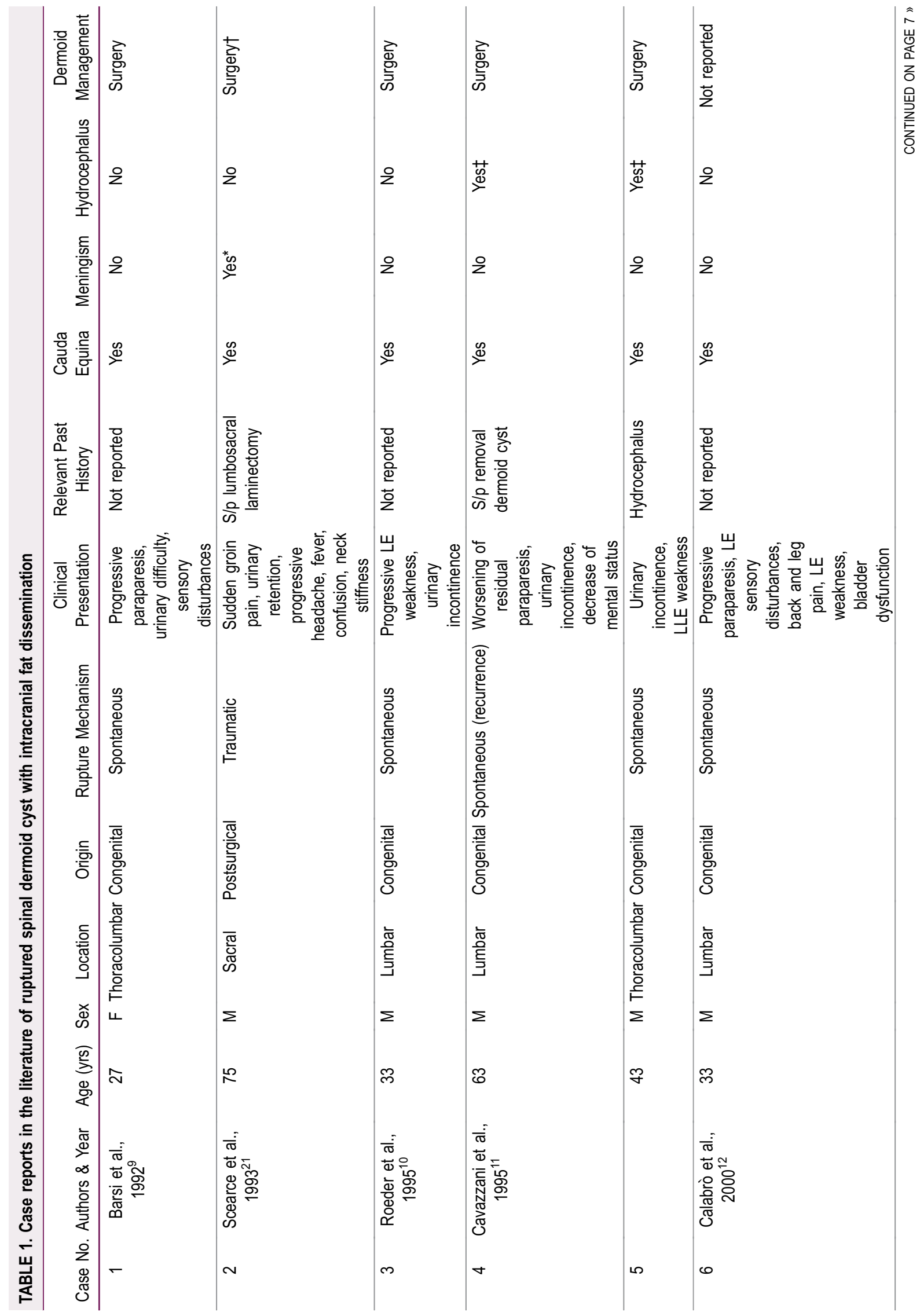




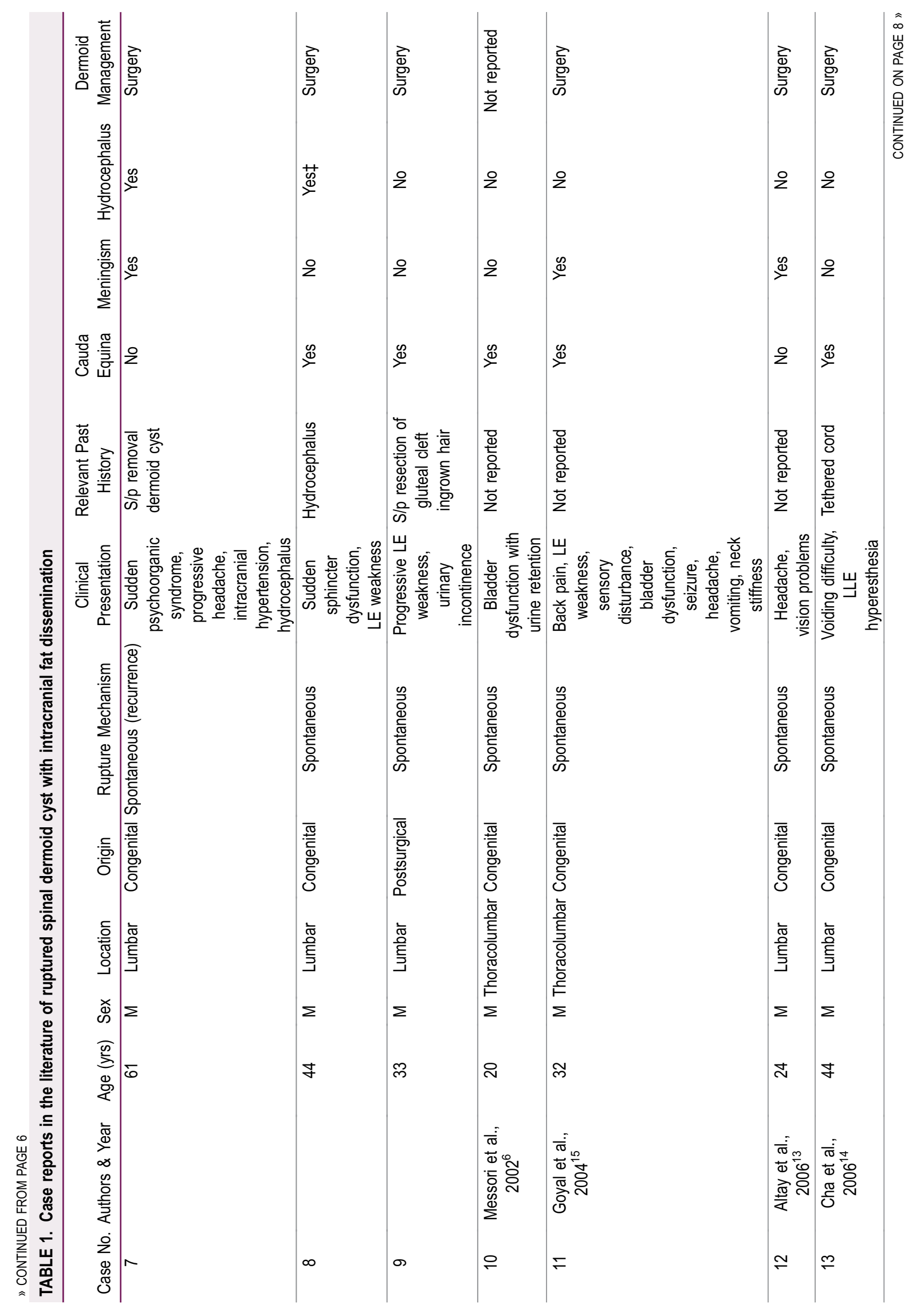

J Neurosurg Case Lessons | Vol 2 | Issue 24 | December 13, 2021 | 7 


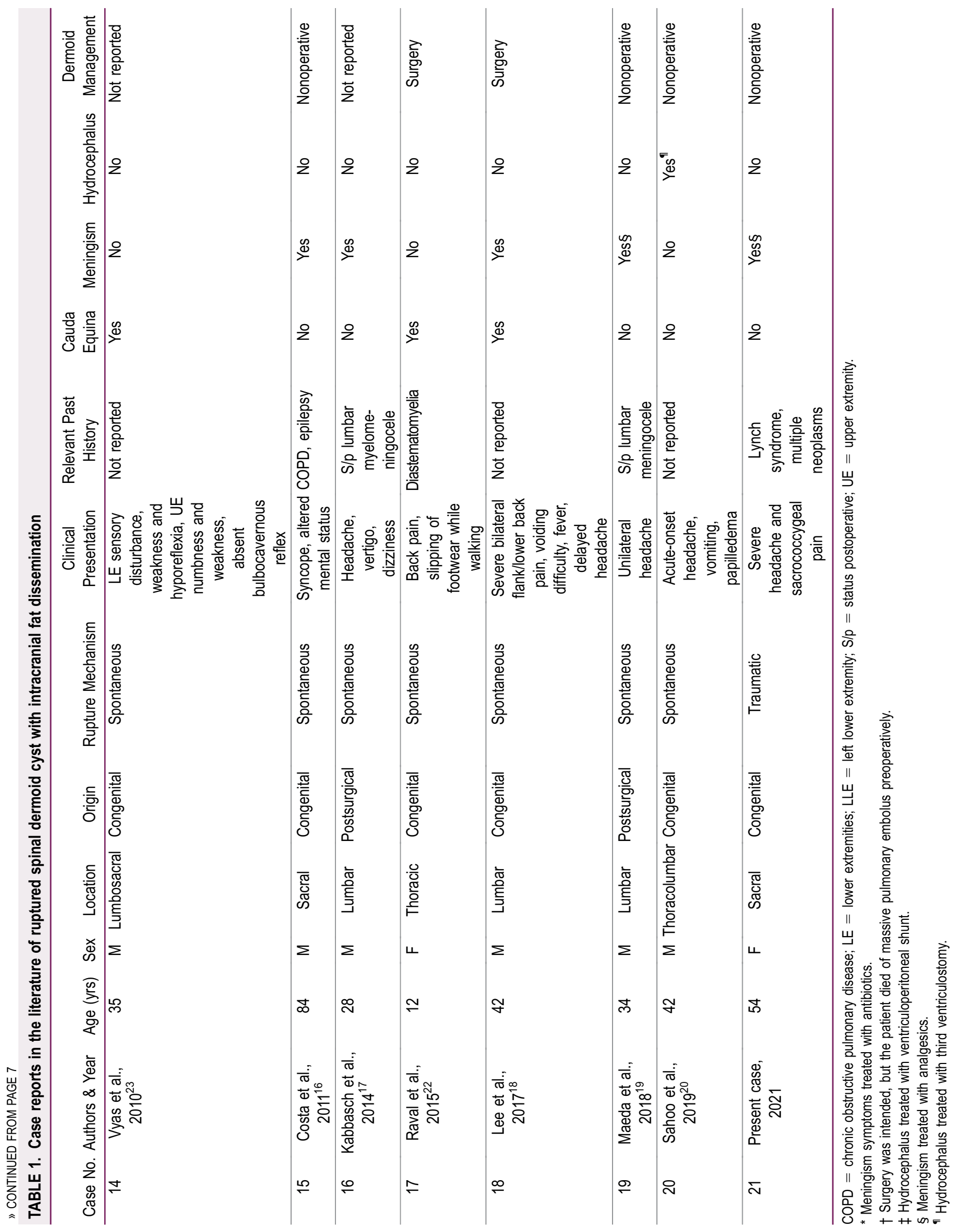


of analgesics to treat this complication to achieve symptom relief, ${ }^{19}$ whereas 1 reported the use of intravenous antibiotics. ${ }^{21}$ However, the administration of systemic steroids in this context should also be considered because it has been reported to ameliorate the transient chemical meningitis symptoms experienced after intracranial dermoid cyst rupture. ${ }^{27-29}$ In patients who developed hydrocephalus, CSF diversion was the rule despite the spinal dermoid cyst having been diagnosed or not. Most patients with hydrocephalus underwent ventriculoperitoneal shunting, ${ }^{11,12}$ but in one case, a third ventriculostomy was performed. ${ }^{20}$ Due to the life-threatening nature of hydrocephalus, an urgent CSF diversion procedure is unquestionable, regardless of what surgery would be performed.

Unfortunately, the current literature about this topic has several limitations. Several articles did not explicitly report what management was pursued after diagnosis of the spinal dermoid cysts (i.e., observation versus surgery), and many articles did not mention what therapy was administered when meningism symptoms were present. Furthermore, complications or long-term follow-up after a given management were not mentioned in most reports. However, despite those limitations, the analysis of the current available literature still favors the complete surgical removal of the spinal dermoid cyst in this clinical scenario.

\section{Lessons}

Intraspinal dermoid cyst rupture must be considered when intracranial subarachnoid or intraventricular fat is discovered on intracranial imaging. Ruptured dermoid cysts can present with cauda equina syndrome, meningism, or acute hydrocephalus. Although management of ruptured spinal dermoid cysts must be decided on an individual basis, definitive surgical treatment is usually favored due to the risk of complications from either acute rerupture or slow progression. After removal, close follow-up is required due to risk of recurrence. CSF diversion must be prioritized when hydrocephalus is present, and either analgesia or steroids can be recommended to treat meningism symptoms.

\section{Acknowledgments}

We extend our sincere thanks to Stella Seal for her invaluable support in developing the search strategy.

\section{References}

1. Abramson RC, Morawetz RB, Schlitt M. Multiple complications from an intracranial epidermoid cyst: case report and literature review. Neurosurgery. 1989;24(4):574-578.

2. Arseni $C$, Dănăilă L, Constantinescu $A$, Carp N. Spinal dermoid tumours. Neurochirurgia (Stuttg). 1977;20(4):108-116.

3. Bailey I. Dermoid tumours of the spinal cord. $J$ Neurol Neurosurg Psychiatry. 1967;30(6):588-589.

4. Falavigna A, Righesso O, Teles AR. Concomitant dermoid cysts of conus medullaris and cauda equina. Arq Neuropsiquiatr. 2009;67(2A):293-296.

5. Gatam L, Merthana PE. A rare case of intramedullary spinal dermoid cyst in adult: a case report. Int J Surg Case Rep. 2020;73:52-57.

6. Messori A, Polonara G, Serio A, Gambelli E, Salvolini U. Expanding experience with spontaneous dermoid rupture in the MRI era: diagnosis and follow-up. Eur J Radiol. 2002;43(1):19-27.

7. Zhang $Y$, Cheng JL, Zhang L, et al. Magnetic resonance imaging of ruptured spinal dermoid tumors with spread of fatty droplets in the central spinal canal and/or spinal subarachnoidal space. J Neuroimaging. 2013;23(1):71-74.
8. Garg K, Kumar R. Isolated central canal rupture of spinal dermoid. Report of 3 cases. J Neurosurg Spine. 2014;21(3):361-366.

9. Barsi P, Kenéz J, Várallyay G, Gergely L. Unusual origin of free subarachnoid fat drops: a ruptured spinal dermoid tumour. Neuroradiology. 1992;34(4):343-344.

10. Roeder MB, Bazan C, Jinkins JR. Ruptured spinal dermoid cyst with chemical arachnoiditis and disseminated intracranial lipid droplets. Neuroradiology. 1995;37(2):146-147.

11. Cavazzani P, Ruelle A, Michelozzi G, Andrioli G. Spinal dermoid cysts originating intracranial fat drops causing obstructive hydrocephalus: case reports. Surg Neurol. 1995;43(5):466-470.

12. Calabrò $F$, Capellini $C$, Jinkins JR. Rupture of spinal dermoid tumors with spread of fatty droplets in the cerebrospinal fluid pathways. Neuroradiology. 2000;42(8):572-579.

13. Altay $\mathrm{H}$, Kitiş $\mathrm{O}$, Calli $\mathrm{C}$, Yünten $\mathrm{N}$. A spinal dermoid tumor that ruptured into the subarachnoidal space and syrinx cavity. Diagn Interv Radiol. 2006;12(4):171-173.

14. Cha JG, Paik SH, Park JS, Park SJ, Kim DH, Lee HK. Ruptured spinal dermoid cyst with disseminated intracranial fat droplets. $\mathrm{Br} \mathrm{J}$ Radiol. 2006;79(938):167-169.

15. Goyal A, Singh D, Singh AK, Gupta V, Sinha S. Spontaneous rupture of spinal dermoid cyst with disseminated lipid droplets in central canal and ventricles. J Neurosurg Sci. 2004;48(2):63-65.

16. Costa F, Robino AM, Crespi S, Fornari M, Palmieri G. Syncope revealing a ruptured sacral dermoid cyst in an 84-year-old man: case report. J Neurosurg Sci. 2011;55(3):289-292.

17. Kabbasch C, Dorn F, Mpotsaris A, Weber C, Liebig T. Rupture of a spinal dermoid cyst may lead to dissemination and progress of Fatty tissue in the central spinal canal and intracranial subarachnoid space. A case report. Neuroradiol J. 2014;27(6):759-763.

18. Lee HC, Choi I, Lim YM, Jang MU, Kim JY, Kang SY. Delayed severe headache due to ruptured spinal dermoid cyst. Headache. 2017;57(4):668-669.

19. Maeda K, Inoue A, Hirano S, Sugihara Y. Lipid droplets in the basal cistern and ventricles. Intern Med. 2018;57(19):2913-2914.

20. Sahoo SK, Dhandapani S, Wankhede L. Wax droplets lining ventricles. World Neurosurg. 2019;128:206-208.

21. Scearce TA, Shaw CM, Bronstein AD, Swanson PD. Intraventricular fat from a ruptured sacral dermoid cyst: clinical, radiographic, and pathological correlation. Case report. J Neurosurg. 1993;78(4):666-668.

22. Raval M, Purohit S, Mishra V, Shah T. Ruptured thoracic intraspinal dermoid cyst in a patient with skeletal abnormalities of thoracic spine - a case report. Egypt J Radiol Nucl Med. 2015;46(2):435-439.

23. Vyas S, Giragani S, Singh P, Khandelwal N. Ruptured spinal dermoid with central canal and intraventricular extension. Neurol India. 2010;58(4):678-679.

24. Gagnier JJ, Kienle G, Altman DG, Moher D, Sox H, Riley D; CARE Group. The CARE guidelines: consensus-based clinical case reporting guideline development. BMJ Case Rep. 2013;2013:bcr2013201554.

25. Moher D, Liberati A, Tetzlaff J, Altman DG; PRISMA Group. Preferred reporting items for systematic reviews and meta-analyses: the PRISMA statement. Int J Surg. 2010;8(5):336-341.

26. McGowan J, Sampson M, Salzwedel DM, Cogo E, Foerster V, Lefebvre C. PRESS peer review of electronic search strategies: 2015 guideline statement. J Clin Epidemiol. 2016;75:40-46.

27. Esquenazi Y, Kerr K, Bhattacharjee MB, Tandon N. Traumatic rupture of an intracranial dermoid cyst: case report and literature review. Surg Neurol Int. 2013;4:80.

28. Kim IY, Jung S, Jung TY, Kang SS, Kim TS. Traumatic rupture of an intracranial dermoid cyst. J Clin Neurosci. 2008;15(4):469-471.

29. Carvalho GA, Cervio A, Matthies C, Samii M. Subarachnoid fat dissemination after resection of a cerebellopontine angle dysontogenic cyst: case report and review of the literature. Neurosurgery. 2000;47(3):760-764. 


\section{Disclosures}

Dr. Witham reported other from Augmedics outside the submitted work. Dr. Bettegowda reported consultant from DePuy Synthes and consultant from Bionaut Labs outside the submitted work. The remaining authors report no conflict of interest concerning the materials or methods used in this study or the findings specified in this paper.

\section{Author Contributions}

Conception and design: Theodore, Perdomo-Pantoja, Zakaria, Porras, Azad, Witham, Bettegowda. Acquisition of data: Theodore, PerdomoPantoja, Zakaria, Porras, Azad, Hwang. Analysis and interpretation of data: Perdomo-Pantoja, Zakaria, Judy, Khalifeh, Porras, Hwang,
Bettegowda. Drafting the article: Theodore, Perdomo-Pantoja, Judy, Khalifeh. Critically revising the article: Theodore, Zakaria, Judy, Khalifeh, Porras, Azad, Witham, Bettegowda. Reviewed submitted version of manuscript: Theodore, Zakaria, Judy, Khalifeh, Porras, Azad, Hwang, Witham. Approved the final version of the manuscript on behalf of all authors: Theodore. Statistical analysis: Perdomo-Pantoja. Administrative/technical/material support: Theodore, Porras, Bettegowda. Study supervision: Zakaria, Azad, Bettegowda.

\section{Correspondence}

Nicholas Theodore: Johns Hopkins University School of Medicine, Baltimore, MD. theodore@jhmi.edu. 